\title{
A Responsive Framework for Optimal Advertising Policy in the Digital Music Market
}

\author{
Tobey H. Ko and Henry Y. K. Lau \\ Department of Industrial and Manufacturing Systems Engineering, The University of Hong Kong, Pokfulam, Hong \\ Kong \\ Email: \{magicfor, hyklau \}@hku.hk
}

\begin{abstract}
This paper proposes a novel responsive framework to help the record label in making profit maximizing advertising decisions in the product pre-release phase. We describe a market of potential consumers where the consumer behaviors are backed by an interactive Markov Chain, in which the periodic advertising activities carried out by the record label varies the population frequencies of the potential consumer market. We demonstrate the working of the proposed framework through an illustrative numerical example, results of the study show the proposed framework's superiority both in terms of reach to potential consumers and the net profit it brings.
\end{abstract}

Index Terms - interactive Markov chain, music industry, decision support, optimal advertising.

\section{INTRODUCTION}

The entertainment industry is a billion dollar business. The industry is not only characterized by the hypes and creativity featured in its products, the enormous amount of investment required to commit prior to the release or even production of these products, not to mention each one of the product is also sold at an extremely low price compared to its required initial investment, so that an extremely large quantity is needed to be sold in order for the investors to make a profit or just to break even. It is obvious that, behind the shiny objects shown in the entertainment industry, the creators and investors often have to make decisions under huge risk. Even after the product is in its finished form, the marketing and promotion process prior to the release of these products are crucial to the success and profitability of the investment, as the marketing process is what draws the audience to the entertainment products. The objective of the paper is to provide a conceptual framework for decision support in the pre-release marketing and promotion stage for entertainment products. The model intends to provide a responsive advertising decisionmaking framework under budget and time constraints for the industry to help them maximize potential profit upon release of the products.

Among different sectors in the entertainment industry, the music industry is particularly prominent to the associated high investment risk. Given the entertainment

Manuscript received February 5, 2015; revised May 26, 2015. products' experience goods nature and the ease of accessing the musical products, investing in the music industry has never been more risky with the tougher competitive market and the increasing threat in recent years with the development of free music streaming platforms. However, being known as in the creative industry, use of scientific methods in forecasting and optimizing the market outcomes are rarely practiced in the decision-making processes in the industry. As a result, decisions are very often made based on experience or intuition, thus there's little assurance for a fair return on investment in this industry, and some potentially profitable products may not be able to live up to their promises due to the lack of proper decision-making tools. The objective of the proposed model is to assist the decision-makers to dynamically vary their traditional advertising policy to adapt to the changing populations in each behavioral states, in order to maximize the reach to consumers at the end of the promotional period. An interactive Markov Chain is employed to model the change of behavior states among consumers, and the intensity of word-of-mouth effect would have a strong impact towards the transition probability between different behavioral states.

The proposed framework is largely inspired by literature in two important streams: application of social contagion in the field of word-of-mouth marketing, and the revenue forecasting model for entertainment products.

The study of social contagion, or sometimes called social learning, has been around for over half a decade. Prior to the popularity of online social networking, the studies of social contagion have been focused on adoption of innovation and new technologies. The most well-known study was the work by Ryan and Gross [1], Griliches [2] and Hagerstrand [3] in the phenomenon of slow adoption of hybrid corn seeds among the corn farmers. Their research on the seemingly irrationally slow adoption rate has resulted in the discovery of spatial patterns in the diffusion process. Such pattern displays a slow initial rate of adoption, followed by the adoption of the hybrid corn seed by the adopted farmers' neighbors, then the adoption process slowly picked up as more of the neighbors joined the club of hybrid corn seed adopters. Similar study regarding the adoption of new drug or medicine by physicians or doctors for a given city of population in Coleman et al. [4], [5], and Valente [6] have all yield comparable results. The study of word-of- 
mouth (WOM) marketing extends the early research on social contagion theory into the decision-making process of the consumers. First pioneered by Katz and Paul in [7] with a survey on "personal influence" for other sampled members in pursuit of a set of daily activities. Incorporating the concepts of word-of-mouth marketing and the consumers' activities over the internet, later studies conducted by Godes and Mayzlin [8], Chevalier and Mayzlin [9], Zhang and Dellarocas [10], and Liu [11] all show a significant link between word-of-mouth activities and the sales of the products in their studies.

Owing to the empirically proven effectiveness of word-of-mouth marketing in prior studies, we observe a recent trend of applying methodologies in discovering word-of-mouth effects into the revenue / performance forecasting models for various consumer products. Although little has been done in the music industry, revenue forecasting and decision-support models on the motion pictures draw very close to our problem on hand. In particular, the neural networks-based prediction model by Sharda and Delen [12], and the diffusion models proposed by Dellarocas, Zhang and Awad [13] have all shown promising high forecasting accuracy of the movies' box-office performance using online reviews and word-of-mouth marketing. However, most of the studies in forecasting motion picture's box office performance are focusing on the word-of-mouth effects after the release of the motion picture. Our proposed framework, a pre-release advertising and promotion decision framework in essence, is largely influenced by the the Markov-based decision support system introduced by Eliashberg, Jonker, Sawhney, and Wierenga [14], in which their view on the dynamic interaction between potential consumers in different behavior states is particularly applicable to the market for digital music, where a lot of songs climb to the top of sales chart due to consumers actively sharing the song in their online social network.

Our paper proposes a dynamic feedback control system on top of a revenue forecasting model which adapts to external shocks in the potential consumer market. The fact that the booming online social networks can create unexpected online sensations and bestsellers in a matter of hours supports our view of a more dynamic market and the need for deployment of a control system. The rest of the paper introduces the proposed responsive framework in detail, and present a simple numerical example to showcase the effectiveness of the model. Finally, the practical implications of the proposed framework are discussed, and suggestions for future research are given.

\section{PROPOSED RESPONSIVE FRAMEWORK}

The proposed framework is a resource constrained discrete-time stochastic model aimed at helping the record labels to optimally adapt their advertising policy with respect to the market response over a fixed promotional period to maximize the profit from the sale of musical products at the end of the promotional period. The model builds on the behavior representation of the music consumers' learning and cognition process for a particular music album over a fixed promotional period, mimicking the on-line word-of-mouth process popularized in recent years. At any given time in the fixed promotional period, a music consumer can be found in one of the state defined in the behavior representation, and the state transitions are modeled using an interactive Markov Chain.

\section{A. Behavior Representation}

The proposed framework partitions the market of potential consumers into 5 mutually exclusive behavioral states: unaware, aware, immune, forgotten, and cognition. Depending on the marketing and advertising policy of the record label, the market of potential consumers are exposed to information shared within the consumption network, and the proportion of consumers in each state evolves along with the variation in advertising policy. A more detailed description for each behavioral states are listed below:

- S1 - Unaware: Consumers who are unaware of the existence of the new musical product.

- S2 - Aware: Consumers who are aware of the existence of the new musical product through marketing informational exposure or word-ofmouth message relayed within the consumption network, but have not decided to further relay (share) the information in the network.

- S3 - Immune: Consumers who are aware of the existence of the new musical product through marketing informational exposure or word-ofmouth message relayed within the consumption network, but decided to ignore the product due to a mis-match between the consumer's and the product's characteristics.

- S4 - Forgotten: Consumers who were aware of the existence of the new musical product through marketing informational exposure or word-ofmouth message relayed within the consumption network, but no longer respond to any messages in the network regarding the product due to a lack of repeated interaction with the product information.

- S5 - Cognition: Consumers who are aware of the existence of the new musical product through marketing informational exposure or word-ofmouth message relayed within the consumption network, and are actively relaying (sharing) the messages within the network.

\section{B. State Transitions}

The music consumers' behavioral representation and the respective state transitions are depicted in Fig. 1. At the beginning of the promotional period, all consumers are assumed to be in the Unaware state, because none of the consumers would be getting any information about the musical product at this instance. As the promotion kicks off, the consumers may, at any discrete time within the fixed promotional period, be exposed to marketing and advertising information put forth by the record label or relayed by fellow consumers within the network. The information exposure $\left(\mathrm{IE}_{1}\right)$ is an intermediate event in the model, it is assumed only when the consumers getting the 
information exposure can a state change be occurred. Consumers who have reached the intermediate information exposure event may experience a change of behavior state depending on the effectiveness of information exposure as well as the consumers' own taste preference. Even if the information exposure is effective, there may still be a portion of consumers switching to Immune state due to a taste mismatch.

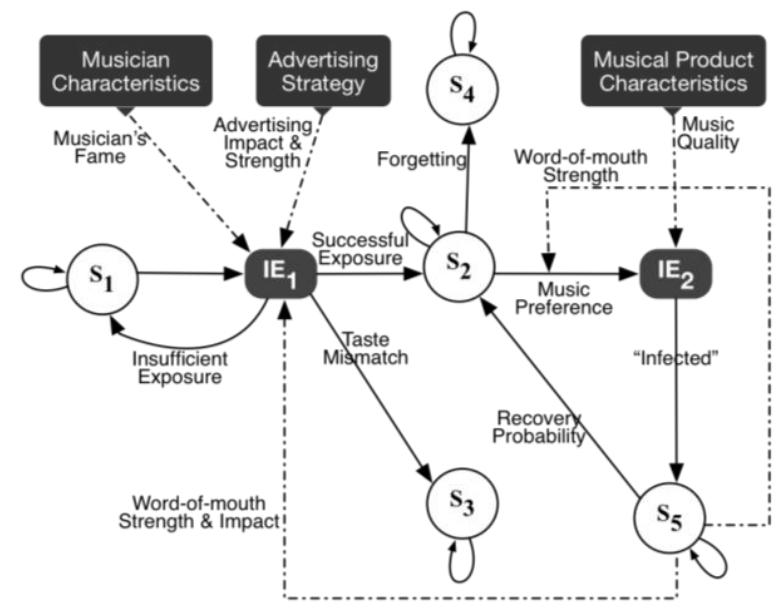

Figure 1. Behavior representation in market for digital music

At any subsequent periods after the beginning of the promotion, consumers who are in the Aware state may go through further state change as a result of repeated exposure to word-of-mouth messages. The Aware consumers may go through an intermediate event of music sampling $\left(\mathrm{IE}_{2}\right)$ to gather more information (for example, to learn the quality of the musical product), and the portion of consumers who are impressed after the sampling process would proceed to transition to the Cognition state and begin to share and relay word-ofmouth message within the network. In the case that the repeated exposure to word-of-mouth messages relayed within the network did not trigger the music sampling intermediate event, the consumer may stay in the Aware state or transition to the Forgotten state if the probability of repeated exposure to word-of-mouth messages falls too low.

It is assumed the consumers who are in the Immune and Forgotten state will not go through further state transitions, while those in the Cognition state may stay in the state or transition back to the Aware state overtime.

\section{Interactive Markov Chain}

The proposed framework models the state transitions in the behavioral representation using a discrete statespace discrete time interactive Markov Chain. As described in the state transitions section above, the need to model the state transitions as an interactive Markov Chain (as opposed to a standard Markovian model) arises from the need of interaction between consumers in different states. For instance, by incorporating the wordof-mouth interaction process into the consideration of information exposure and repeated exposure, the Cognition consumers play a vital role in the state transitions as their interaction with the Unaware and
Aware consumers would alter the probability of state transitions for these consumers.

The basic premise of the proposed framework model builds on the standard Markovian property where at time $t$, the vector population frequencies for the next period (at time $t+1)$ is independent of the history of population frequencies and would only depend on the vector population frequencies in the current period. In such case, the vector of population frequencies for $t+1$ is,

$$
Q_{t+1}=P_{t} Q_{t}
$$

where $\mathrm{Q}_{\mathrm{t}}=\left[\mathrm{q}_{\mathrm{it}}\right]$ denotes the vector of population frequencies for each state $i$ at time $t$, and $P_{t}=\left[P_{i j t}\right]$ is a vector representing all possible state transition probabilities from states $i$ to states $j$ at time $t$.

Extending the assumed behavior of a standard Markovian model, we incorporate element of social interaction among individual consumers into the state transition consideration of consumers in certain states in the proposed model using the concept of interactive Markov Chain proposed by Conlisk [15]. In his 1976 paper, Conlisk [15] introduced a generic interactive Markov chain model where the state transition probabilities $\mathrm{P}_{\mathrm{t}}$ is allowed to vary depend on the distribution of population across different state spaces. Under this assumption, the interactive Markov chain carries a property that:

$$
Q_{t+1}=P\left[Q_{t}\right] Q_{t}
$$

in which $P[\cdot]$ can be used to represent a variety of social interactions. Considering the proposed framework models a process of promoting message of a certain product (a piece of music album in this case), the word-of-mouth promotion process is similar to a viral diffusion process in a social network, we draw inspiration from network diffusion models when formulating the interactive Markov chain.

1) Population frequency specification: Here we give a detailed account for the specifications of the proposed interactive Markov chain model with respect to the behavior representations in Fig. 1. With reference to the standard Markovian model in (1), we denote the vector of population frequencies at time $t\left(\mathrm{Q}_{\mathrm{t}}\right)$ as

$$
Q_{t}=\left[\begin{array}{lllll}
U_{t} & A_{t} & I_{t} & F_{t} & C_{t}
\end{array}\right]^{T}
$$

where:

$U_{t}=$ Unaware state's population frequency at time t;

$A_{t}=$ Aware state's population frequency at time $\mathrm{t}$;

$I_{t}=$ Immune state's population frequency at time $\mathrm{t}$;

$F_{t}=$ Forgotten state's population frequency at time t;

$C_{t}=$ Cognition state's population frequency at time $\mathrm{t}$.

It is obvious that since each individual consumer would only be in 1 state at any time instance, the state population frequencies should be mutually exclusive from each other, and they would also sum up to the entire population of the potential consumer market (equal to one).

2) Model parameters: The state transition probabilities are governed by a number of parameters in the proposed model, they are: 
$M_{F}$ : Musician's fame. Probability that a randomly selected potential consumer will be made aware of the musician's new musical product after exposure due to the musician's prior achievements.

$M_{Q}$ : Music quality. Probability that a randomly selected potential consumer in the Aware state will find the musical product attractive after sampling, and will start recommending the product through word-of-mouth messages over the internet.

$I_{M t}$ : Advertising impact factor at time $t$. The probability that an exposure to the advertising would lead to a state transition from Unaware to Aware.

$P[M]_{t}$ : Advertising exposure probability at time $t$. The probability for being exposed to advertisements between time period $[t, t+1]$.

$\lambda_{t}$ : Word-of-mouth frequency at time $t$. Rate parameter for the word-of-mouth spreading process. Assumed to follow an exponential distribution.

$I_{W t}$ : Word-of-mouth impact factor at time $t$. Probability that an exposure to the word-of-mouth message would lead to a state change from Unaware to Aware.

$\rho$ : Taste-matching probability. Probability that a randomly selected potential consumer would accept the musical product's genre or theme after exposure.

$\theta$ : Sampling probability. Probability that an Aware consumer is willing to sample the musical product due to a general liking to music.

$\delta$ : Recovery probability. Probability that a randomly selected potential consumer in the Cognition state will experience a state change from Cognition to Aware.

$\beta$ : Forgetting probability. Probability that a randomly selected potential consumer in the Aware state will experience a state change from Aware to Forgotten in case no sampling nor word-of-mouth exposure takes place.

3) Transition probabilities: According to Fig. 1, the transition probabilities $P_{t}$ is:

$$
P_{t}=\left[\begin{array}{ccccc}
p_{U U t} & p_{U A t} & p_{U I t} & 0 & 0 \\
0 & p_{A A t} & 0 & p_{A F t} & p_{A C t} \\
0 & 0 & 1 & 0 & 0 \\
0 & 0 & 0 & 1 & 0 \\
0 & p_{C A t} & 0 & 0 & p_{C C t}
\end{array}\right]^{T}
$$

where $p_{U U t}$ refers to the state transition probability for the fraction of population in the Unaware state at time $t$ to remain in the Unaware state at time $t+1, p_{U A t}$ refers to the state transition probability for the fraction of population in the Unaware state at time $t$ to transition to the Aware state at time $t+1$, and so on. The formulation of these state transition probabilities are discussed below.

With accord to the Interactive Markov Chain Property from Conlisk [15], the word-of-mouth exposure initiated by the population frequencies in the Cognition state during the time period $[t, t+1]$ is modeled as:

$$
P[W]_{t}=\left(1-e^{-\lambda_{t}}\right) C_{t}
$$

where $\lambda$ is the rate of word-of-mouth in which the population of Cognition consumers actively sharing the messages of the said musical product within the network, and $C_{t}$ is the population frequency in the Cognition state at time $t$.

In the proposed interactive Markov Chain model, the exposure to word-of-mouth messages effects in parallel to the information exposure put forth by the record label's marketing and advertising effort, thus the Unaware consumers may be exposed to both types of exposure during any time within the promotional period. However, given the information exposure is an intermediate event, only the proportion of Unaware population that have information exposure may be able to go through state transition to either Aware or Immune, we can therefore define the transition probability $P_{U U t}$ as follows:

$$
\begin{aligned}
P_{U U t}= & 1-\left[P[M]_{t}+P[W]_{t}\right]+ \\
& {\left[\rho M_{F}\left(\left(1-I_{M t}\right) P[M]_{t}+\left(1-I_{W t}\right) P[W]_{t}\right)\right] }
\end{aligned}
$$

The state transition probability from Unaware to Unaware shown in (6) is divided into 2 parts: the probability that the Unaware consumers is not made available to any form of information exposure (first line of the equation), and the probability that the Unaware consumer is not impacted by the information exposure to go through further state transition (second line of the equation).

By the same logic, the state transition probabilities for $P_{U I t}$ and $P_{U A t}$ are constructed as follows:

$$
\begin{gathered}
P_{U I t}=(1-\rho)\left[P[M]_{t}+P[W]_{t}\right] \\
P_{U A t}=1-P_{U U t}-P_{U I t}
\end{gathered}
$$

Refer to the transition probabilities in (6) - (8), it is important to note that the musician's fame $M_{F}$ only affects the transition probability to the Aware state but not the transition probability to the Immune state. This setting gives a more realistic touch to the model, as consumers who are drawn to specific genres of music would not pay attention to music that's outside of his/her musical taste regardless of the fame of the musician whom produces that piece of musical product.

The population frequency in the Aware state also has 3 transition probabilities: $P_{A F t}, P_{A C t}$, and $P_{A A t}$. The transition probabilities are defined as follows:

$$
\begin{gathered}
P_{A F t}=\beta\left[\left(\left(1+P[W]_{t}\right)(1-\theta)\right)\left(1-P[W]_{t}\right)\right] \\
P_{A C t}=\left(1+P[W]_{t}\right) \theta M_{Q} \\
P_{A A t}=1-P_{A F t}-P_{A C t}
\end{gathered}
$$

The transition probability from Aware to Forgotten shown in (9) puts on specific restriction to Aware consumers to forget the given musical product. For instance, the Aware consumer will only be subjected to the forgetting probability $\beta$ if he/she have no exposure to the word-of-mouth message, and have not performed any form of sampling during the period $[t, t+1]$. For a state transition from Aware to Cognition, the Aware consumer is required to undergo a sampling intermediate event. According to (10), although the word-of-mouth exposure $P[W]_{t}$ increases the probability of sampling, the quality of the musical product $M_{Q}$ is vital to whether the 
consumer will be "infected" by the sample and start spreading the word-of-mouth message in the next period in Cognition state.

The transition probabilities $P_{C A t}$ and $P_{C C t}$ for population frequency in the Cognition state are rather straightforward:

$$
\begin{gathered}
P_{C A t}=\delta \\
P_{C C t}=1-\delta
\end{gathered}
$$

The transition probabilities for population frequencies in the Cognition state are not influenced by the properties of the interactive Markov Chain. As a result, only the recovery probability $\delta$ will affect the population changing state from Cognition to Aware.

\section{Responsive Model for Optimal Advertising Policy}

The purpose of the responsive model is to assist record labels to make better promotional decisions which also adapt to the dynamics in the market, the changes in the vector of population frequencies to be exact.

Assuming the population willing to make a purchase for the given musical product at the end of the promotional period is directly proportional to the population frequencies in the Aware state and in the Cognition state at time $\mathrm{T}$, the goal of the profitmaximizing record label during the promotional period is to maximize the reach to music consumers in the market, so that the combined population frequencies in the Aware state and the Cognition state at time $\mathrm{T}$ is maximized. Therefore, the record label would, at the beginning of each promotional period, decide on the optimal level of promotional activity for that period, subjected to a fixed promotional budget for the entire promotional period.

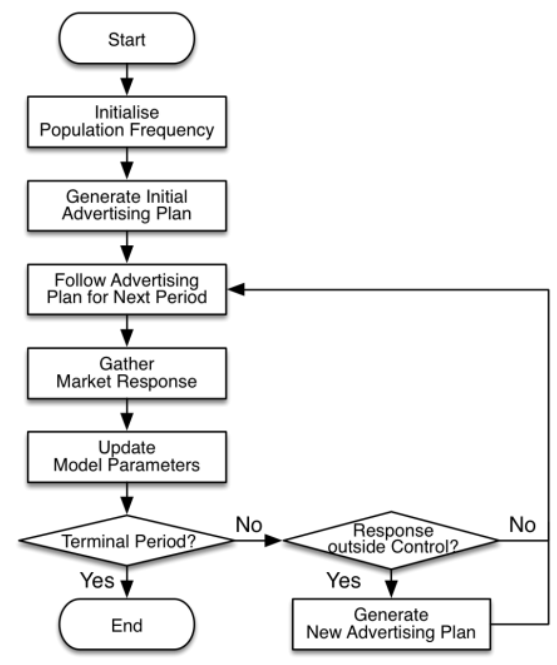

Figure 2. Control flow of the proposed responsive model

The control flow in Fig. 2 is responsible for generating responsive advertising plans for the record label to maximize the reach to potential consumers during the promotional period. To follow the logic of the responsive model, the record label first gathers the population frequencies in a given consumer market and an initial advertising plan is generated accordingly. The advertising plan will be followed through during the promotional period until the gathered market response from advertising is outside of a said control limit predetermined by the record label, in which case a new advertising plan will be generated for the remaining time in the promotional period.

The advertising plan, although aimed at maximizing population frequencies in the Aware and Cognition states, is essentially a profit maximizing model intended to maximize the record label's profit in selling the musical product after the promotional period. Thus, we formulate the profit maximization advertising model as

$$
\begin{aligned}
& \prod_{0}\left(M_{F}, M_{Q}\right)= \\
& \max _{1, \ldots, T} \quad\left\{(1+r)^{-T} \cdot g_{T}\left(N_{T}, \lambda_{T}, M_{Q}, p\right)\right. \\
&\left.-\sum_{t=1}^{T}(1+r)^{-t} \cdot M_{t}\left(Q_{t-1}, p_{t-1}\right)\right\}
\end{aligned}
$$

where,

$$
N_{T}=C_{T}+A_{T}
$$

s.t.

$$
\sum_{t=1}^{T} M_{t} \leq B
$$

In (14), the marketing and advertising plan for the given musical product will be decided to maximize the discounted net profit from sale of the musical product at the end of the promotional period. Assuming the given musical product is priced at $p$, the expected profit from sale of the musical product $g_{T}$ is discounted back to the start of the promotional period at $t=0$ using the interest rate $r$. The marketing and advertising expenditure for each period $\left(M_{t}\right)$ are discounted using the interest rate $r$ and subtracted from the discounted profit to yield the discounted net profit to the record label. The profit maximizing advertising model in (14) is used to generate the initial advertising plan, as well as the new advertising plans whenever necessary. If a new advertising plan is needed, the updated model parameters gathered from the market responses will be used in generation of the new plan, thus making the model more responsive to the dynamics in the market.

\section{NUMERICAL EXAMPLE}

We employ a numerical example to demonstrate how the proposed responsive framework works in context.

\section{A. Setting of Numerical Example}

The numerical example uses the following fictional setting as shown in Table I to test out its effectiveness:

Refer to Table I, we consider an advertising campaign in a market with population size $(\mathrm{G})$ of 1000 , where the interest rate is at $5 \%$ and each unit of musical product is priced at $p=10$ dollars. The record label has a budget of $\$ 1000$ dollars which can be spent across a 10-period promotional period. For the sake of simplicity, many of the model parameters are pre-defined and kept constant throughout, the record label only have to choose the level 
of advertising for each period. There are altogether 4 levels of advertising expenses to choose from: high $\left(M_{t}^{H}\right)$, medium $\left(M_{t}^{M}\right)$, low $\left(M_{t}^{L}\right)$, and no advertising $\left(M_{t}^{0}\right)$, where each level of these advertising expenditure brings a different advertising exposure probability $\left(P[M]_{t}^{H}\right.$, $P[M]_{t}^{M}, P[M]_{t}^{L}$, and $P[M]_{t}^{O}$ respectively) to influence the population frequencies of the interactive Markov Chain. Half way through the simulation of the numerical example, an external shock is introduced which decreases the word-of-mouth frequency from $\lambda_{1}$ to $\lambda_{6}$ after the end of period 5. This shock triggers the responsive model to generate a new profit maximizing advertising plan under the new model parameter, the results of the numerical example simulation is shown in Table II.

TABLE I. SETTINGS For NumericAl EXAMPLE

\begin{tabular}{|c|c|c|c|c|}
\hline \multicolumn{5}{|c|}{ Model Parameters } \\
\hline$M_{F}$ & $M_{Q}$ & $I[M]_{t}$ & $\lambda_{I}$ & $\lambda_{6}$ \\
\hline 0.5 & 0.5 & 0.3 & 0.5 & 0.1 \\
\hline$I_{W t}$ & $\rho$ & $\theta$ & $\beta$ & $\delta$ \\
\hline 0.3 & 0.8 & 0.8 & 0.1 & 0.1 \\
\hline \multicolumn{5}{|c|}{ Advertising Variables } \\
\hline$B$ & $M_{t}^{H}$ & $M_{t}^{M}$ & $M_{t}^{L}$ & $M_{t}^{0}$ \\
\hline 1000 & 200 & 100 & 50 & 0 \\
\hline$T$ & $P[M]_{t}^{H}$ & $P[M]_{t}^{M}$ & $P[M]_{t}^{L}$ & $P[M]_{t}^{0}$ \\
\hline 10 & 0.5 & 0.2 & 0.1 & 0 \\
\hline \multicolumn{5}{|c|}{ Model Constants } \\
\hline & $r$ & $G$ & $p$ & \\
\hline & 0.05 & 1000 & 10 & \\
\hline
\end{tabular}

TABLE II. RESUltS For NUMERICAL EXAMPLE

\begin{tabular}{|c|c|c|c|}
\hline \multicolumn{5}{|c|}{ Advertising Plans } \\
\hline Periods & $P_{B}$ & $P_{I}$ & $P_{N}$ \\
\hline 1 & 100 & 0 & 0 \\
\hline 2 & 100 & 0 & 0 \\
\hline 3 & 100 & 0 & 0 \\
\hline 4 & 100 & 0 & 0 \\
\hline 5 & 100 & 200 & 200 \\
\hline 6 & 100 & 200 & 200 \\
\hline 7 & 100 & 0 & 200 \\
\hline 8 & 100 & 200 & 200 \\
\hline 9 & 100 & 200 & 200 \\
\hline 10 & 100 & 200 & 0 \\
\hline \multicolumn{5}{|c|}{ Advertising Results } \\
\hline$N_{T}$ & $58.26 \%$ & $66.22 \%$ & $66.47 \%$ \\
\hline$\Pi_{0}$ & 2657 & 3372 & 3359 \\
\hline
\end{tabular}

\section{B. Results for Numerical Example}

Refer to Table II, the benchmark plan $\left(\mathrm{P}_{\mathrm{B}}\right)$ used in the numerical simulation employs medium level of advertising every week, whereas both the initial advertising plan $\left(\mathrm{P}_{\mathrm{I}}\right)$ and the new advertising plan have scheduled the advertising starting mid-way of the promotional period. Comparing the results of the advertising campaigns, the proposed profit maximizing advertising model out-performs the benchmark advertising plan both in terms of net profit and reach to potential consumers. Furthermore, the responsive framework has displayed a capability to adjust the advertising strategy when the market environment has changed during the advertising campaign. Specifically, in the numerical example, the new advertising plan $\left(\mathrm{P}_{\mathrm{N}}\right)$ responses to a drop in word-of-mouth frequency by scheduling the advertising activities earlier.

Fig. 3 shows a comparison of the growth of consumer population under different advertising plans used in the numerical example. Compared to the steady increase of consumer population resulted from the benchmark advertising plan, the plans generated using the proposed advertising model start rather late in the promotional period, yet the stronger advertising intensity brings in consumer population at a much faster pace and end up both surpassing the effects of the benchmark advertising plan.

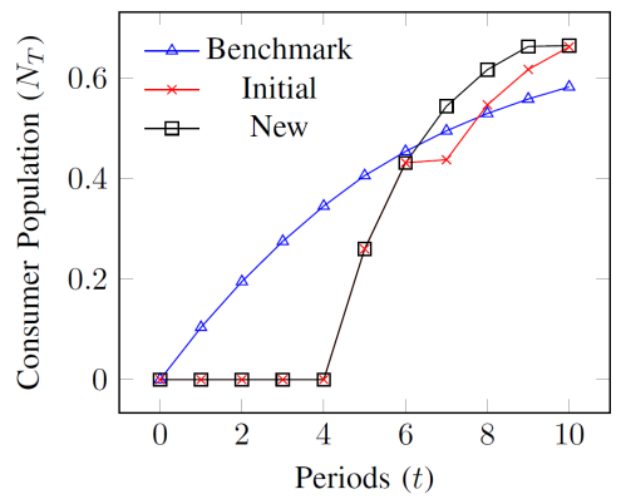

Figure 3. Variations in consumer population $\left(\mathrm{N}_{\mathrm{T}}\right)$

\section{Practical Implications}

The numerical example demonstrates the proposed responsive framework's potential in assisting the producers to make promotional decisions in a market with word-of-mouth activities. In particular, the framework acts as a toolkit to help the producers effectively time their promotional activities, in order to generate the greatest promotional impact in the consumer market. Besides, the control system incorporated into the proposed framework can effectively help the decision makers to react to market disturbances; by scheduling promotional activities earlier when observing the market reacting poorly to the previous promotions, and delay the activities when the market is already in the hype, the proposed framework can be deployed to help producers to respond to market dynamics automatically.

\section{CONCLUSION}

This paper presents a novel interactive Markov Chainbased responsive framework for planning of marketing and advertising activities to promote a musical product which goes on sale at the end of a promotional period. It contributes to the growing amount of literature on decision support systems in the internet era. The proposed framework presented in this paper has shown its superiority in making profit maximizing scheduling decisions in a market with dynamically changing 
environment. Alternatively, the proposed framework can also be used to assist the producers to more accurately time their new product release when the promotional period constrain is lifted. The proposed responsive framework can be further extended to make marketing and advertising decisions after product release, where the market is more dynamic and decisions affect a longer time period.

\section{REFERENCES}

[1] B. Ryan and N. C. Gross, "The diffusion of hybrid seed corn in two Iowa communities," Rural Sociol., vol. 8, no. 1, pp. 15-24, 1943.

[2] Z. Griliches, "Hybrid corn: An exploration in the economics of technological change," Econom. J. Econom. Soc., pp. 501-522, 1957.

[3] T. Hagerstrand, "Innovation diffusion as a spatial process," Innov. Diffus. Spat. Process, 1968.

[4] J. Coleman, E. Katz, and H. Menzel, "The diffusion of an innovation among physicians," Sociometry, vol. 20, no. 4, pp. 253270, 1957.

[5] J. Coleman, E. Katz, and H. Mentzel, Medical Innovation: Diffusion of a Medical Drug Among Doctors, Indianap, MN: Bobbs-Merrill, 1966.

[6] T. W. Valente, "Social network thresholds in the diffusion of innovations," Soc. Netw., vol. 18, no. 1, pp. 69-89, 1996.

[7] E. Katz and F. Paul, Personal Influence: The Part Played by People in the Flow of Mass Communications. New York: Free Press, 1955.

[8] D. Godes and D. Mayzlin, "Using online conversations to study word-of-mouth communication," Mark. Sci., vol. 23, no. 4, pp. $545-560,2004$

[9] J. A. Chevalier and D. Mayzlin, "The effect of word of mouth on sales: Online book reviews," J. Mark. Res., vol. 43, no. 3, pp. 345$354,2006$.

[10] X. Zhang and C. Dellarocas, "The lord of the ratings: Is a movie's fate is influenced by reviews?" in Proc. ICIS 2006, 2006, pp. 117.

[11] Y. Liu, "Word of mouth for movies: Its dynamics and impact on box office revenue," J. Mark., vol. 70, no. 3, pp. pp. 74-89, 2006.

[12] R. Sharda and D. Delen, "Predicting box-office success of motion pictures with neural networks," Expert Syst. Appl., vol. 30, no. 2, pp. 243-254, Feb. 2006.
[13] C. Dellarocas, X. (Michael) Zhang, and N. F. Awad, "Exploring the value of online product reviews in forecasting sales: The case of motion pictures," J. Interact. Mark., vol. 21, no. 4, pp. 23-45, 2007.

[14] J. Eliashberg, J. J. Jonker, M. S. Sawhney, and B. Wierenga, "MOVIEMOD: An implementable decision-support system for prerelease market evaluation of motion pictures," Mark. Sci., vol. 19, no. 3, pp. 226-243, Jul. 2000.

[15] J. Conlisk, "Interactive markov chains," J. Math. Sociol., vol. 4, no. 2, pp. 157-185, Jan. 1976.

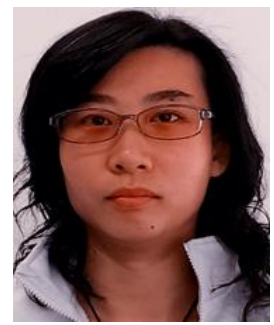

Tobey H. Ko received the B.Eng degree in industrial engineering and technology management and the B.BA degree in finance from the University of Hong Kong, in 2011 and 2012, respectively. She is currently working towards the Ph.D. degree at the Department of Industrial and Manufacturing Systems Engineering, University of Hong Kong, Hong Kong.

Her research interests include operations research and decision support system for digital information goods.

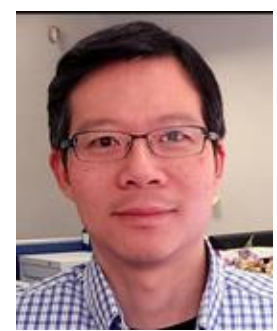

Henry Y. K. Lau is an Associate Professor in the Department of Industrial and Manufacturing Systems Engineering, The University of Hong Kong. Henry graduated from the University of Oxford with a BA Degrees in Engineering Science and a DPhil in Robotics. Prior to joining The University of Hong Kong, he has been working in industry for many years as a Systems Engineer and Section Manager at the UK Atomic Energy Authority (UKAEA) and AEA Technology plc., working on projects involving bespoke tele-robotics systems and advanced automation systems for the nuclear industry in decommissioning and waste management. While working in England, Henry was a Croucher Foundation Research Fellow at the University of Oxford Robotics Research Group, and a visiting lecturer at Brasenose College teaching Engineering Science. Henry's research interest includes artificial intelligence, in particular in artificial immune systems (AIS), intelligent automation for material handling, virtual and augmented reality systems. 